\title{
EFECTIVIDAD DE LA REACCIÓN EN CADENA DE LA POLIMERASA EN TIEMPO REAL (PCR-RT) E INMUNODOT EN EL DIAGNÓSTICO TEMPRANO DE LEPTOSPIROSIS: ANÁLISIS COMPARATIVO CON LA PRUEBA DE MICROAGLUTINACIÓN (MAT)
}

\section{Effectiveness of the real-time polymerase chain reaction (RT-PCR) and Immunodot in the early diagnosis of Leptospirosis: comparative analysis with the Microagglutination test (MAT)}

\author{
María Zunilda Núñez ${ }^{\mathrm{a}}$, María Lucía Fortuna ${ }^{\mathrm{b}}$, Bienvenido Veras ${ }^{\mathrm{c}}$, Aseret Medi-nad, Luis \\ Mena ${ }^{\mathrm{e}}$, Elizabeth Gutiérrez ${ }^{f}$, Cristobalina Ramírez ${ }^{\mathrm{g}}$, Coral Vargas ${ }^{\mathrm{h}}$ y Argelia Aybar ${ }^{\mathrm{i}}$
}

Recibido: 26 de abril, $2021 \bullet$ Aprobado: 14 de junio, 2021

Cómo citar: Núñez MZ, Fortuna ML, Veras B, Medina A, Mena L, Gutiérrez E, Ramírez C, Vargas C, Aybar A. Efectividad de la reacción en cadena de la polimerasa en tiempo real (PCR-RT) e Inmunodot en el diagnóstico temprano de leptospirosis: análisis comparativo con la prueba de Microaglutinación (MAT). cysa [Internet]. [citado 22 de febrero de 2022];6(1):17-24. Disponible en: https://revistas. intec.edu.do/index.php/cisa/article/view/2413

\section{Resumen}

Introducción: la leptospirosis es una enfermedad infecciosa producida por espiroquetas del género Leptospira. Se disemina a través de la orina de animales domésticos, con mayor frecuencia por roedores. En República Dominicana se necesitan pruebas confiables para el diagnóstico en etapas tempranas de la enfermedad. En el presente trabajo se aborda la validez diagnóstica del PCR en Tiempo Real y del IGM (INMUNODOT) en comparación con la prueba de Microaglutinación (MAT).

Materiales y métodos: se realizó un estudio observacional, descriptivo, de corte transversal con 69 pacientes admitidos en el Hospital Regional "José María Cabral y Báez"

\footnotetext{
${ }^{a}$ Autor de correspondencia. Especialista en Medicina Interna, Pontificia Universidad Católica Madre y Maestra (PUCMM) y Centro de Investigaciones Biomédicas y Clínicas del Hospital Regional Universitario José María Cabral y Báez. (HRU-JMCB) Santiago, República Dominicana. ORCID: 0000-0001-5386-0117

Correo-e: mz.nunez@ce.pucmm.edu.do.

b Especialista en Medicina Interna. (PUCMM) y (HRU-JMCB). ORCID: 0000-0002-1778-9621, Correo-e: marialucia4tuna@gmail.com

c Doctor en Medicina, (HRUJMCB). ORCID: 0000-0003-1515-8997, Correo-e: bienvenido.veras@ucateci.edu.do

d Especialista en Medicina Interna. (PUCMM).

ORCID: 000-0003-2720-4727, Correo-e: dra.medinavargas@gmail.com
}

\begin{abstract}
Introduction: Leptospirosis is an infectious disease caused by spirochaetes of the genus Leptospira. It spread through the urine of domestic animals most frequently in rodents. Need reliable tests to diagnose in early stages of the disease and it has been proposed the use of the PCR in real-time as an option. The present work deals with the diagnostic real-time PCR and IGM (INMUNODOT) in comparison with the Microagglutination (MAT) test.

Materials and methods: An observational, descriptive, cross-sectional study with 69 patients admitted as possible Leptospirosis in the Hospital "Jose Maria Cabral y Báez" of Santiago from 2010 to 2012.

\footnotetext{
Doctor en Medicina. (PUCMM) y (HRUJMCB).

ORCID: 0000-0001-7463-2842, Correo-e: 1.mena@ce.pucmm.edu.do ${ }_{\mathrm{f}}$ Especialista en Medicina Interna, (PUCMM) y (HRU-JMCB). ORCID: 0000-0003-1263-8338

Correo-e: elizabethgutierrezmendez@hotmail.com

g Doctora en Medicina, (HRUJMCB). ORCID: 0000-0002-8367-9367, Correo-e: cristobalina.ramirez@hotmail.com

${ }^{\text {h }}$ Doctora en Medicina. (PUCMM) y (HRUJMCB).

ORCID: 0000-0003-4418-1758, Correo-e: coralvargas04@gmail.com

Doctora en Medicina, Especialista en Patología, Magíster en Investigación en Ciencias Médicas. Ph. D. en Ciencias Médicas. Laboratorio de Patología Molecular MEDIPATH. ORCID: 0000-0002-4770-1846, Correo-e: argelia.aybar@gmail.com
} 
con diagnóstico presuntivo de leptospirosis desde el 2010 hasta el 2012

Resultados: del total de los casos (69), la mayoría fueron del sexo masculino $(94.2 \%)$, menores de 49 años $(79.4 \%)$ y provenían de la provincia de Santiago (58\%).

La mortalidad fue de $52.1 \%$ de los cuales $52.3 \%$ fueron reportados positivos para Leptospira y $47.6 \%$ resultaron negativos según el MAT.

Al comparar los resultados de PCR en relación al MAT se obtuvo una sensibilidad de $27.3 \%$ y una especificidad de $80 \%$. Los resultados del PCR y del Immunodot fueron
equivalentes.

Conclusión: en el presente trabajo la realización del PCR en sangre, después del 5 to día de inicio de la enfermedad, no demostró ser mejor que la Inmunodot para la detecno demostró ser mejor que la Inmunodot para la detección temprana de la enfermedad, al contrastarlos con el resultado del MAT. En República Dominicana, los casos hospitalizados con diagnóstico presuntivo de leptospirosis permanecen con muy alta mortalidad. Por tanto, es prioritario optimizar el diagnóstico y el tratamiento de estos casos. En este estudio, los casos confirmados con Leptospirosis que fallecieron indican que debe actualizarse el protocolo de tratamiento y asegurarse que pueda implementarse. Por otra parte, los casos no confirmados fallecidos exigen investigar otras causas de enfermedad como la Infección por Hanta Virus.

Palabras clave: Leptospirosis Humana; PCR; Inmunodot; MA'T

\section{Introducción}

La leptospirosis es una enfermedad infecciosa causada por espiroquetas patógenas del género Leptospira que afecta a humanos y animales. Es una de las zoonosis más comunes del mundo ${ }^{1,2}$. Se disemina a través de la orina de animales domésticos, principalmente roedores. Los factores de riesgo para la infección incluyen: exposición ocupacional (agricultores, ganaderos, trabajadores de mataderos, cazadores, veterinarios, leñadores, trabajadores de alcantarillado, arroceros, comerciantes de mascotas, militares, trabajadores de laboratorio, etc); actividades recrea-
Results: Of the total cases (69), most were male (94.2\%), under 49 years of age $(79.4 \%)$ coming from the province of Santiago (58\%). Mortality was $52.1 \%$ of which $52.3 \%$ were reported positive for leptospirosis and $47.6 \%$ were negative according to the MAT.

To compare the results of PCR in relation to the MAT was obtained a sensitivity of $27.3 \%$ and a specificity of $80 \%$. The results of the PCR and immunodot were equivalent.

Conclusion: In this study, the realization of the PCR in blood after the 5 th day of the disease not proved to be better than the Inmunodot for the early detection of the disease. Is necessary to evaluate cases less than 5th day. In the Dominican Republic, hospitalized with a presumptive diagnosis of Leptospirosis cases remain with very high mortality. Therefore, it is important to optimize the diagnosis and treatment of these cases. In this study, confirmed cases with Leptospirosis who died, indicate that you must upgrade the treatment protocol and ensure that it can be implemented. On the other hand, the deceased not confirmed cases require to investigate other causes of disease as the Hanta Virus infection.

Keywords: Human Leptospirosis; PCR-RT; Inmunodot; MAT

tivas (en agua dulce, ríos, piscinas, canoas, ciclismo de montaña, etc); exposición doméstica (perros, ganado domesticado, sistemas de captación de agua de lluvia, infestación por roedores infectados, etc); u otros (caminar descalzo a través de aguas superficiales, lesiones de piel, contacto con roedores silvestres, exposición accidental, etc.). ${ }^{2-6}$.

La incidencia en el trópico es aproximadamente diez veces mayor que en regiones templadas 7 . Se ha reportado que la incidencia anual oscila entre $0,1 \mathrm{y}$ 1 por cada 100,000 personas residentes en las zonas templadas, a 10 o más por 100,000 en personas que

\section{María Zunilda Núñez, María Lucía Fortuna, Bienvenido Verasc, Aseret Medi-na, Luis Mena, Elizabeth Gutiérrez} Cristobalina Ramírez, Coral Vargas y Argelia Aybar

viven en climas tropicales y hasta 100 o más por cada 100,000 personas cuando ocurren brotes ${ }^{8}$. La magnitud del problema en las regiones tropicales y subtropicales puede atribuirse, en gran medida, a las condiciones climáticas y ambientales?. El riesgo de brotes aumenta durante las temporadas ciclónicas e inundaciones ${ }^{10}$. En el 2007, en la República Dominicana, luego del paso de los huracanes Noel y Olga, se registraron 2,355 casos de leptospirosis, con una tasa de mortalidad de 24.8 por cada 100,000 habitantes. En el 2010, año en el cual no hubo procesos climatológicos de importancia, la tasa de morbilidad y la tasa de mortalidad descendieron a 2.3 y 0.3 por cada 100,000 habitantes, respectivamente ${ }^{11}$. Además, esta enfermedad se reconoce como una causa de fiebre en turistas en los trópicos, incluyendo los visitantes de la República Dominicana ${ }^{12}$.

El diagnóstico adecuado y a tiempo de la leptospirosis es aún problemático ${ }^{12}$. Con frecuencia, esta enfermedad se presenta con síntomas y signos similares a otras enfermedades infecciosas, como dengue, malaria, chikungunya y zika ${ }^{13}$, por lo cual puede confundirse con facilidad. El curso clínico de esta varía desde una presentación asintomática, hasta un cuadro severo con alta mortalidad ${ }^{10}$. La mayoría de los casos son leves y autolimitados o subclínicos, pero algunos pueden complicarse de forma severa y ser potencialmente fatales. La enfermedad tiene un periodo de incubación de 2 a 26 días (en promedio 10 días) y se presenta generalmente con inicio abrupto, contracturas musculares, mialgias y cefaleas en un 75 a $100 \%$ de los pacientes. Otros signos son eyección conjuntival, náuseas, vómitos, diarreas, faringitis, esplenomegalia, linfadenopatía, hepatomegalia, auscultación respiratoria anormal y erupciones en la piel. Generalmente, los hallazgos se atribuyen a la respuesta inmunológica del huésped, más que a la acción directa de la espiroqueta ${ }^{10}$. Algunos autores confirman que existen evidencias sólidas de que los mecanismos inmunopatológicos contribuyen a la evolución de la enfermedad ${ }^{14-16}$.
Aunque los pacientes sospechosos tengan datos de historia de exposición, signos y síntomas sugestivos de leptospirosis y hallazgos de laboratorio que descarten posibles diagnósticos diferenciales, el diagnóstico de la enfermedad debe ser confirmado por pruebas de laboratorios específicas. Las pruebas habituales para leptospirosis son imprecisas y son foco de debate e investigación, particularmente las que podrían ser confirmatorias en las etapas tempranas de la enfermedad. Algunas técnicas moleculares como la Reacción en Cadena de la Polimerasa (PCR, por sus siglas en inglés) se recomiendan para contribuir al diagnóstico en su etapa temprana ${ }^{17,18}$.

Cardona et al., ${ }^{19}$ en un estudio sobre el diagnóstico de leptospirosis mediante PCR en pacientes con síndrome febril icterohemorrágico, encontraron que la PCR tiene un valor predictivo positivo de $40 \% \mathrm{e}$ indicaron que la PCR es capaz de detectar leptospiras en sangre antes de que los anticuerpos séricos puedan ser detectados. Encontraron, además, que la eficiencia de la PCR fue mayor en muestras de orina.

En algunos estudios que evaluaron la sensibilidad y especificidad de las pruebas diagnósticas, se encontró que la sensibilidad del cultivo fue de $10.5 \%$; la del test de microaglutinación (MAT), $49.8 \%$; y la sensibilidad de la combinación de ambas, $55.5 \%$. La PCR por sí sola, fue ligeramente más sensible $(55 \%)$. Sin embargo, la prueba de ensayo de flujo lateral tuvo una sensibilidad de $85.6 \%$ y combinada con la PCR, mostró la mejor sensibilidad y especificidad: $93.2 \%$ y $93.1 \%$, respectivamente. Estos autores sugieren que el uso de MAT subestima la incidencia y prevalencia de la leptospirosis ${ }^{20}$

Numerosas pruebas serológicas alternativas al MAT han sido desarrolladas. Algunos estudios favorecen el uso del IgM dot-ELISA y ELISA IgM como rastreo inicial rápido de la infección por Leptospira, con subsecuente confirmación con MAT ${ }^{19-21}$.

En este sentido, la presente investigación, como objetivo primario, buscó evaluar la efectividad y validez 
diagnóstica de la Reacción en Cadena de Polimerasa en tiempo real (PCR-RT), IgM por Inmunodot y el test de Microaglutinación (MAT) para el diagnóstico temprano en pacientes con sospecha clínica de Leptospirosis Humana. Además, como objetivos secundarios, se buscó determinar la proporción de casos sospechosos de Leptospirosis Humana que tuvieron confirmación del diagnóstico por la prueba de MAT y caracterizar los datos sociodemográficos de los pacientes confirmados con la enfermedad.

\section{Materiales métodos}

Diseño del estudio

Se realizó un estudio observacional, descriptivo, de corte transversal de pacientes admitidos desde el año 2010 hasta el 2012 con diagnóstico presuntivo de leptospirosis en el Hospital Regional Universitario “José María Cabral y Báez" (HRUJMCB) de Santiago de los Caballeros, República Dominicana, para comparar la validez diagnóstica de tres pruebas de laboratorio para Leptospirosis Humana.

Participantes

La población de estudio estuvo compuesta por los adultos que acudían a la emergencia del HRUJMCB con cuadro clínico sugestivo de leptospirosis. Se utilizaron como criterios de inclusión las características clínicas vigentes para realizar un diagnóstico presuntivo de leptospirosis, descritas por las Normas Nacionales de Vigilancia Epidemiológica (fiebre de comienzo repentino, cefalea, escalofríos, mialgia intensa e inyección de conjuntivas). Se excluyeron los pacientes que resultaron positivos para otras enfermedades infecciosas, como Malaria, Hepatitis A, B y C. El estudio contó con la aprobación del Comité de ética del hospital de estudio y del Consejo Nacional de Bioética en Salud (CONABIOS). Se obtuvo un consentimiento o asentimiento informado de todos los pacientes.
Variables

Los parámetros generales que se emplearon para describir la población fueron los datos demográficos (edad, sexo, nacionalidad y lugar de residencia) e información clínica estandarizada para diagnóstico de leptospirosis (signos y síntomas). El diagnóstico de leptospirosis fue confirmado mediante las siguientes pruebas de laboratorio: Reacción en Cadena de Polimerasa en tiempo real (PCR-RT), IgM por Immunodot y el test de Microaglutinación (MAT). Se tomaron las muestras luego del ingreso del paciente al hospital.

La PCR-RT se analizó por medio del kit de extracción de ADN de Tejido de Oiagen y se envió a un laboratorio de Patología Molecular. La IgM Inmunodot se analizó mediante el kit de DST de Inmunodot. Para el test de Microaglutinación (MAT) la muestra fue tomada por el Departamento de Epidemiología del hospital y posteriormente enviada al Laboratorio Veterinario Central (LAVECEN) de la ciudad de Santo Domingo, República Dominicana. El tiempo aproximado para el traslado es de tres horas.

Análisis estadístico

La efectividad de la prueba de PCR-RT e Inmunodot fue evaluada usando los parámetros de sensibilidad, especificidad, valor predictivo positivo y negativo, empleando la prueba de Microaglutinación (MAT) como prueba estándar o "gold standard". Por otra parte, la relación entre la prueba MAT y la mortalidad fue calculada por medio de chi-cuadrado $\left(\mathrm{X}^{2}\right)$, estimando que la asociación tiene significancia cuando el p-valor es $<0.05$.

\section{Resultados}

Se evaluaron 250 pacientes, de los cuales 69 cumplieron con los criterios de elegibilidad. De estos 69, en 6 casos no se obtuvo el resultado del MAT, para un $8,69 \%$ del total de muestras. Se observó

María Zunilda Núñez, María Lucía Fortuna, Bienvenido Verasc, Aseret Medi-na, Luis Mena, Elizabeth Gutiérrez Cristobalina Ramírez, Coral Vargas y Argelia Aybar

que la mayoría de los casos eran hombres $(94.2 \%)$ menores de 49 ańos (79.4\%). El lugar de procedencia más frecuente fue la ciudad de Santiago de los Caballeros (58\%). Los serotipos más frecuentes de Leptospira identificados por la prueba MAT fueron: 1) Leptospira Grippo (18.1 \%); Leptospira pomona (15.1\%); y Leptospira Ictero-haemorragiae con 4 casos $(12.1 \%)$.

Para el análisis de sensibilidad se empleó el test de MAT como prueba de oro y se compararon las pruebas de PCR-RT e Inmunodot.

$\mathrm{Al}$ realizar el test de MAT, 33 pacientes (52.4\%) resultaron positivos para la enfermedad. La prevalencia de la enfermedad según la prueba PCR-RT fue $23.8 \%$, mientras que la sensibilidad fue $27 \%$ (ideal $80 \%$ ), la especificidad de $80 \%$ (ideal $90 \%$ ), el valor predictivo positivo de $60 \%$ (ideal $100 \%$ ) y el valor predictivo negativo de $50 \%$ (ideal $100 \%$ ). La prevalencia de la enfermedad según la prueba de Inmunodot fue de $28.6 \%$ y la sensibilidad fue de $48 \%$ (ideal $80 \%$ ), la especificidad de $93 \%$ (ideal $90 \%$ ), el valor predictivo positivo de $89 \%$ (ideal $100 \%$ ), y el valor predictivo negativo de $62.2 \%$ (ideal $100 \%$ ).

La mortalidad por leptospirosis fue alta; 35 pacientes $(55.5 \%)$ murieron. La relación entre la prueba MAT y la mortalidad no fue significativa $(\mathrm{p}=0.208)$.

\section{Discusión}

El presente estudio muestra que en República Dominicana los casos hospitalizados con diagnóstico presuntivo de leptospirosis presentan una muy alta mortalidad. El hecho de encontrar una alta proporción de pacientes que fallecieron con pruebas para Leptospira negativa sugiere que se debe investigar la posibilidad de otras afectaciones en dichos individuos. Se recomienda realizar la prueba de Hanta Virus, particularmente en pacientes sospechosos para leptospirosis con datos de hemorragia pulmonar y afección renal, puesto que se ha demostrado la relación entre ambas ${ }^{22,23}$.
Al igual que en otros estudios, en el presente trabajo las pruebas para el diagnóstico temprano de la leptospirosis como el PCR-RT e Inmunodot fueron poco efectivas y es necesario implementar o encontrar nuevas pruebas diagnóstica ${ }^{24,25}$. Estudios sugieren mejores resultados utilizando el PCR-RT en orina ${ }^{19}$. En cambio, la prueba que recientemente ha reportado una mayor sensibilidad $(85.6 \%)$ es la prueba de ensayo de flujo lateral y la combinación de esta con el PCR-RT mostró la mayor sensibilidad y especificidad (93.2 \% y $93.1 \%$, respectivamente $)^{20}$.

Hallazgos recientes con la prueba de Leptocheck-WB (prueba de IgM basada en inmunocromatografía) muestran una alta sensibilidad y aseguran que es efectiva para realizar el diagnóstico de leptospirosis de manera rápida y fácil ${ }^{26}$. Con estos hallazgos, pareciera que la opción ideal para confirmar o descartar la enfermedad podría ser la realización de la prueba de Leptocheck-WB para tamizaje, realización de la prueba de flujo lateral y PCR-RT para confirmación y el test de MAT para identificación de las cepas de leptospirosis.

\section{Conclusiones}

Considerando la alta morbimortalidad en los casos sospechosos de leptospirosis ingresados en hospitales públicos de la República Dominicana y la deficiencia demostrada de pruebas diagnósticas confirmatorias en etapas tempranas de la enfermedad, se justifica continuar investigando sobre este tópico en el país.

Es necesario aumentar los esfuerzos para optimizar el diagnóstico y el tratamiento en casos de Leptospirosis Humana en República Dominicana, mediante la actualización del protocolo de manejo y la garantía de su implementación. El Servicio Nacional de Salud necesita contar con laboratorios modernos para el diagnóstico confirmatorio de la leptospirosis en las diferentes regiones del país, para con esto agilizar y garantizar fiabilidad de los resultados del diagnóstico. Además, se hace necesario caracterizar otras causas de cuadros clínicos similares a la leptospirosis en el país, como, por ejemplo, los causados 
por Hantavirus, considerando el hecho de los casos afectados que mostraron resultados negativos del test de MAT y que manifestaron alteraciones renales y pulmonares. También se sugiere restablecerse la y pulización de autopsias en los pacientes fallecidos realización de autopsias en los pacientes fallecidos
por la enfermedad, para un mejor entendimiento de por la enferm

Por otra parte, recomendamos profundizar en el tema con más investigaciones sobre la asociación entre la aparición de las complicaciones y la mortalidad por leptospirosis con las variables demográficas, el tipo de Leptospira y las alteraciones de laboratorio.

\section{Financiación}

El presente estudio fue realizado con el auspicio del Fondo de Incentivo a la Investigación Científica y Tecnológica (FONDOCYT) del Ministerio de Educación Superior, Ciencia y Tecnología (MESCYT) de la República Dominicana.

\section{Referencias bibliográficas}

1. Gompf S. Leptospirosis: Practice Essentials, Background, Pathophysiology [Internet]. Emedicine. medscape.com. 2021 [citado 10 mayo 2021]. Disponible en: http://emedicine.medscape.com/ Disponible en: http://em
article/220563-overview

Lau C, Townell N, Stephenson E, van den Berg D, Craig S. Leptospirosis: An important zoonosis acquired through work, play and travel. Australian Joumal of General Practice. 2018,47(3):105-10. Do: 10.31128/AFP-07$17-4286$

3. Dunay S, Bass J, Stremick J. Leptospirosis: a Global Health Burden in Review. Emergency Medicine: Open Access. 2016;6(5). Doi: 10.41 72/2165-7548.1000336

4. Wasiński B, Dutkiewicz J. Leptospirosis--current risk factors connected with human activity and the environment. Annals of Agriculture and Environmental Medicine, 2013; 20:239.
5. Abdul Mutalip M, Mahmud M, Lodz N, Yoep N, Muhammad E, Ahmad A et al. Environmental risk factors of leptospirosis in urban settings: a systematic review protocol. BMJ Open, 2019;9(1):e023359. Doi: 10.1136/ bmjopen-2018-023359

6. Biscornet L, Dellagi K, Pages F, Bibi J, de Comarmond J, Melade J, et al. Human leptospirosis in Seychelles: a prospective study confirms the heavy burden of the disease but suggests that rats are not the main reservoir. PLoS Neg Trop Dis, 2017;1(8):e0005831

7. Munoz-Zanzi C, Groene E, Morawski BM, Bonner K, Costa F, Bertherat E, et al. A systematic literature review of leptospirosis outbreaks worldwide, 1970-2012. Rev Panam Salud Pública, 2020;44:e78. Doi: 10.26633/ RPSP.2020.78

8. World Health Organization. Leptospirosis Burden Epidemiology Reference Group (LERG) [Online]. Who.int. 2021 Disponible en: https://www.who.int/zoonoses/diseases/lerg/ en/ [Citado 8 de mayo 2021].

9. World Health Organization. Human leptospirosis: guidance for diagnosis, surveillance and control [Internet]. Apps.who.int. 2021. [Online]. Disponible en: https://apps.who. int/iris/handle/10665/42667 [Citado 5 mayo 2021]

10. Uptodate. Microbiology, epidemiology, clinical manifestations, and diagnosis of leptospirosis. [Online]. Disponible en: https://www. uptodate.com/contents/leptospirosis-epidemiology-microbiology-clinical-manifestations-and-diagnosis\#:- :text=Clinically\%20 apparent $\% 20$ leptospirosis $\% 20$ presents $\% 20$ with,of\%20leptospirosis\%20(picture\%202). [Citado 7 de mayo 2021]
11. Pan American Health Organization. Health in the Americas+, 2012 Edition. Summary: Regiona Outlook and Country Profiles. Washington, D.C.: PAHO; 2012

12. Ricaldi JN, Swancutt MA, Matthias MA. Current trends in translational research in leptospirosis. Curr Opin Infect Dis, 2013;26(5):399-403. Doi: 10.1097/QCO.0000000000000001.

13. De Francesco DE, de Carvalho G, de Sousa Soares D, Mendes M, Parente Filho S, Rocha $\mathrm{H}$, et al. Changing patterns in leptospirosis: a three-decade study in Brazil. Intern J Infect Dis, 2017;60:4-10. Doi: 10.1016/j.ijid.2017.04.023

14. Wagenaar JF, Gasem MH, Goris MG, et al. Soluble ST2 levels are associatedwith bleeding in patients with severe leptospirosis. PLoS Negl Trop Dis, 2009;3:e453. Doi: 10.1371/ journal.pntd.0000453.

15. Croda J, Neto AN, Brasil RA, et al. Leptospirosis pulmonary haemorrhage syndrome is associated with linear deposition of immunoglobulin and complement on the alveolar surface. Clin Microbiol Infect, 2010;16:593-9. Doi: 10.1111/j.1469-0691.2009.02916.x.

16. Nally JE, Chantranuwat C, Wu XY, et al. Alveolar septal deposition of immunoglobulin and complement parallels pulmonary hemorrhage in a guinea pig model of severe pulmonary leptospirosis. Am J Pathol, 2004; 164:1115-27. Doi: 10.1016/S0002-9440(10)63198-7.

17. Waggoner JJ, Pinsky BA. Molecular diagnostics for human leptospirosis. Curr Opin Infect Dis, 2016;29(5):440-5. Doi:10.1097/ QCO.0000000000000295

18. Mullan S, Panwala TH. Polymerase chain reaction: an important tool for early diagnosis of leptospirosis cases. J of Clin and Diag Res:
JCDR, 2016;10(12):DC08-11. Doi: 10.7860/ JCDR/2016/22462.9010.

19. Cardona MN, Moros RM, López EA, Perez JL, Hernández RC. Diagnóstico de leptospirosis mediante la PCR en pacientes con síndrome febril icterohemorrágico, Instituto Nacional de Higiene "Rafael Rangel". Caracas: Ministerio del Poder Popular para la Salud; 2008.

20. Blanco RM, Romero EC. Efficacy of serum samples stored on filter paper for the detection of antibody to Leptospira spp. by microagglutination test (MAT). J Immunol Methods, 2012;386:31-3. Doi: 10.1016/j. jim.2012.08.013.

21. Vanasco NB, Jacob P, Landolt N, Chiani Y, Schmeling MF, Cudos C, et al. Diagnostic accuracy of an IgM enzyme-linked immunosorbent assay and comparison with 2 polymerase chain reactions for early diagnosis of human leptospirosis. Diag Microb and Infect Dis, 2016;84:292-7. Doi: 10.1016/j.diagmicrobio.2016.01.002

22. Kaya S, Yılmaz G, Aydın M, et al. The "MICE" scoring system in differentiating the identical twins leptospirosis and hantavirus infection. Infection, 2020:48:99-107. Doi: 10.1007/ s15010-019-01366-7

23. Bakelants E, Peetermans W, Lagrou K, Meersseman W. Clinical and biochemical differences between hantavirus infection and leptospirosis: a retrospective analysis of a patient series in Belgium. Act Clin Belg, 2020;75(3):185-92. Doi:10.1080/17843286.2019.1591654

24. Winslow WE, Merry DJ, Pirc ML, Devine PL. Evaluation of a commercial enzyme-linked immunosorbent assay for detection of immunoglobulin $\mathrm{M}$ antibody in human leptospiral infection. J Clin Microbiol, 1997;35:1938. Doi:10.1128/JCM.35.8.1938-1942.1997. 
Efectividad de la reacción en cadena de la polimerasa en tiempo real (PCR-RT) e Inmunodot en el diagnóstico temprano de leptospirosis: análisis comparativo con la prueba de Microaglutinación (MAT)

25. Tansuphasiri U, Deepradit S, Phulsuksombati $\mathrm{D}$, Tangkanakul W. Two simple immunoassays using endemic leptospiral antigens for serodiagnosis of human leptospirosis. Southeast Asian J Trop Med Public Health, 2005; 36:302.

26. Eugene EJ, Handunnetti SM, Wickramasinghe SA, Kalugalage TL, Chathuraka R, Wickremesinghe $\mathrm{H}$, et al. Evaluation of two immunodiagnostic tests for early rapid diagnosis of leptospirosis in Sri Lanka: a preliminary study. BMC Infect Dis, 2015;15:319. Doi: 10.1186/ s12879-015-1080-z
27. Bencosme SA, Núñez MZ, Arvelo E, Contreras F, Robledo M. La necropsia y la calidad de los servicios médicos hospitalarios. Correlación clínico-patológica de las causas de muerte de pacientes adultos en el HRUJMCB entre octubre 1979 y abril de 1993. Acta Médica Dominicana, 1996;18(6):224-42; y 1997;19 (1):12-38.

24 | Ciencia y Salud 2022; 6(1, enero-abril): 17-24 • Artículo original 